\title{
Effect of Ambient Reynolds Number on Small Wind Turbine Subjected to Low Wind Speed Conditions
}

\author{
Joel Mbwiga $^{1^{*}}$, Cuthbert Z. Kimambo ${ }^{2}$ and Joseph Kihedu ${ }^{2}$ \\ ${ }^{1}$ Department of Energy and Production, Mbeya University of Science and Technology, Mbeya, \\ Tanzania (*jmbwiga@gmail.com). \\ ${ }^{2}$ Department of Mechanical and Industrial Engineering, University of Dar es Salaam, Dar es \\ Salaam, Tanzania.
}

\begin{abstract}
Wind flow over the airfoil surface is adversely affected by the differences between the design and ambient values of a dimensionless quantity called Reynolds number. Wind turbine designed for high Reynolds Number shows lower maximum power performance when installed in low-speed wind regime. Tanzanian experience shows that some imported modern wind turbines depict lower power performance compared to the drag-type locally manufactured wind turbines. The most probable reason is the difference between design and local ambient Reynolds numbers. The turbine design parameters have their properties restricted to the range of Reynolds numbers for which the turbine was designed for. When a wind turbine designed for a certain range of Reynolds numbers is made to operate in the Reynolds number out of that range, it behaves differently from the embodied design specifications. The small wind turbine of higher Reynolds number will suffer low lift forces with probably occasional stalls.
\end{abstract}

Keyword: Low Reynolds Number, Boundary Layer, Laminar Separation Bubbles.

\section{INTRODUCTION}

The fluid mechanics encompassing wind flow over the aerofoil lies at the foot of geometrical shape of the airfoil surfaces (Kim and Al-Abadi, 2016). The shape modifies the wind flow velocities and pressures over the airfoil surfaces (Mathew, 2004; Manwell et al., 2009). The airfoil geometrical shape is designed such that there is a negative differential pressure at the upper surface in relation to the lower surface and hence a lift force normal to the surface is created (Mathew, 2004; Manwell et al., 2009; Schaffarczyk, 2014). Likewise, as wind flows from leading to trailing edge there is positive pressure gradient due to skin friction which tries to decelerate the flow and hence a drag force against the flow is created (Mathew, 2004; Manwell et al., 2009; Schaffarczyk, 2014). The pressure gradient depends on the chord length, wind velocity and surface roughness which could be summarized into one dimensionless quantity called Reynolds number (Re). As wind blows over the airfoil surface, a boundary layer few millimeters between the surface and free wind stream is formed due to skin friction (Mathew, 2004; Manwell et al., 2009; Schaffarczyk, 2014). Reynolds number characterizes the wind flow in the boundary layer (Fig 1). Reynolds number is defined as Momona Ethiopian Journal of Science (MEJS), V12(2):223-231, 2020 @CNCS, Mekelle University, ISSN:2220-184X Submitted on: $27^{\text {th }}$ May 2019 
the ratio of inertia force due to wind flow to viscous force due to skin friction which could mathematically be expressed as follows (Mathew, 2004; Manwell et al., 2009; Schaffarczyk, 2014; Sabri et al., 2017):

$R e=\frac{\text { Inertial force }}{V \text { iscous force }} \quad R e=\frac{\rho V C}{\mu}=\frac{V C}{v}$

Where,

$v$ is kinetic viscosity,

$\mu$ is dynamic viscosity,

$\mathrm{V}$ is wind speed,

$\mathrm{C}$ is the chord length.

In preliminary design, for simplicity, Reynolds number could be approximated by using the following formula:

$R e=V * C * 70000$

Where,

$\mathrm{V}$ is design wind speed (relative velocity) $[\mathrm{m} / \mathrm{s}]$,

C is design chord length [m] and 70000 is a constant value for air $\left[\mathrm{s} / \mathrm{m}^{2}\right]$ (Hepperle, 2018).

Having estimated the average wind speed in the wind regime, Reynolds number will vary with the chord length. If the chord lengths are not constant, the mean length is assumed.

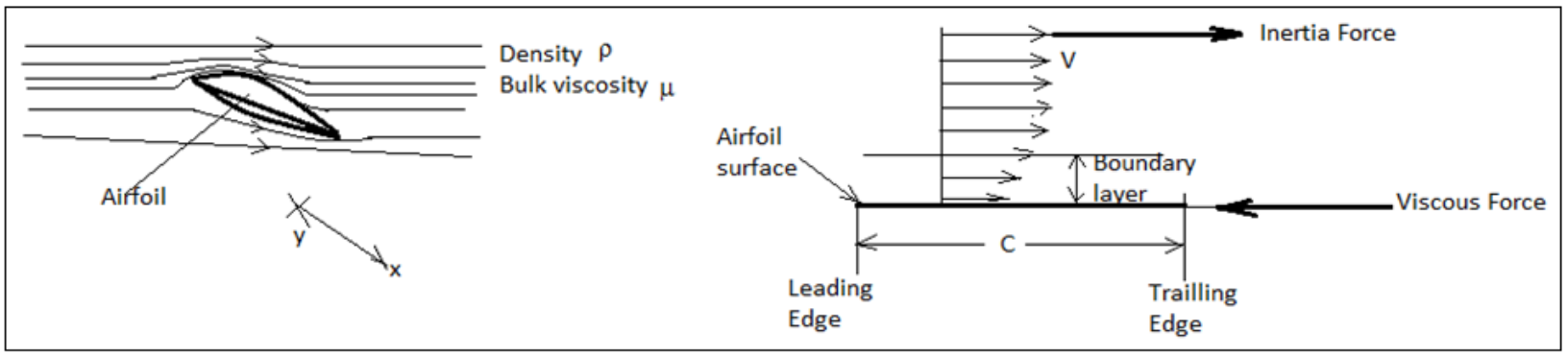

Figure 1. Wind flow passed airfoil profile.

\section{WIND FLOW OVER AIRFOIL SURFACE}

The wind flow in the boundary layer can be either laminar or turbulent depending on Reynolds number. However, the demarcating Reynolds number is not very clear. For internal flow such as flow in a pipe, laminar occurs when Reynolds number is less than 2000 whereas for turbulent flow Reynolds number is greater than 4000 (Sabri et al., 2017; Incropera et al., 2007). For external flow, Laminar occurs up to when Reynolds number equals $5 \times 10^{5}$ (Incropera et al., 2007). For simplicity, 
the threshold value to transition region is taken as $5 \times 10^{5}$ (Shah et al., 2014). For airfoil, free surface flow is presumed, airflow in the boundary layer is considered to be fully laminar up to $1 \times 10^{5}$, between $1 \times 10^{5}$ and $5 \times 10^{5}$ the flow is considered to be in transition to turbulent flow (Yousefi and Razeghi, 2018). In practical depending on the surface roughness the transition range may extend to between $1 \times 10^{5}$ and $3 \times 10^{6}$ (Yousefi and Razeghi, 2018). The critical Reynolds number is found to vary with the geometrical shape of the airfoil. Yousefi and Razeghi (2018) in their experiment on NACA 0012, 0015 and 0018 airfoils found the lowest critical Reynolds number to be $1 \times 10^{5}$, $5 \times 10^{5}$ and $3.5 \times 10^{5}$ respectively.

When the pressure gradient along airfoil surface is adverse such that viscous force becomes equal to or greater than inertia force, the boundary layer separates from the airfoil surface resulting in the decline of lift forces and the increase in drag forces, a condition called Laminar Separation Bubbles (LSB) (Bertin and Cummings, 2009; Rehman et al., 2018; Giguere and Selig, 1997). This condition is undesirable because the wind turbine rotor decelerates or stops immediately and increased drag force results into severe blade vibration imposing a fatigue loading on the rotor blade. As per figure 2, vortices are formed when viscous force exceeds the inertial force. A designer has to strive to reduce the effect of LSB or eliminate its possibility to occur completely. In airplanes, the occurrence of LSB does not give a pilot enough time before realizing an abrupt loss in lift force thereby endangering the airplane and passengers. Though turbulent flow is found to have increased drag which increases fuel consumption of airplane, designers prefers to design the airplane to operate in turbulent to avoid LBS from occurring. Turbulence flow has high stability against separation because of its tendency of flow mixing strengthens the boundary layer (Jahanmiri, 2011).

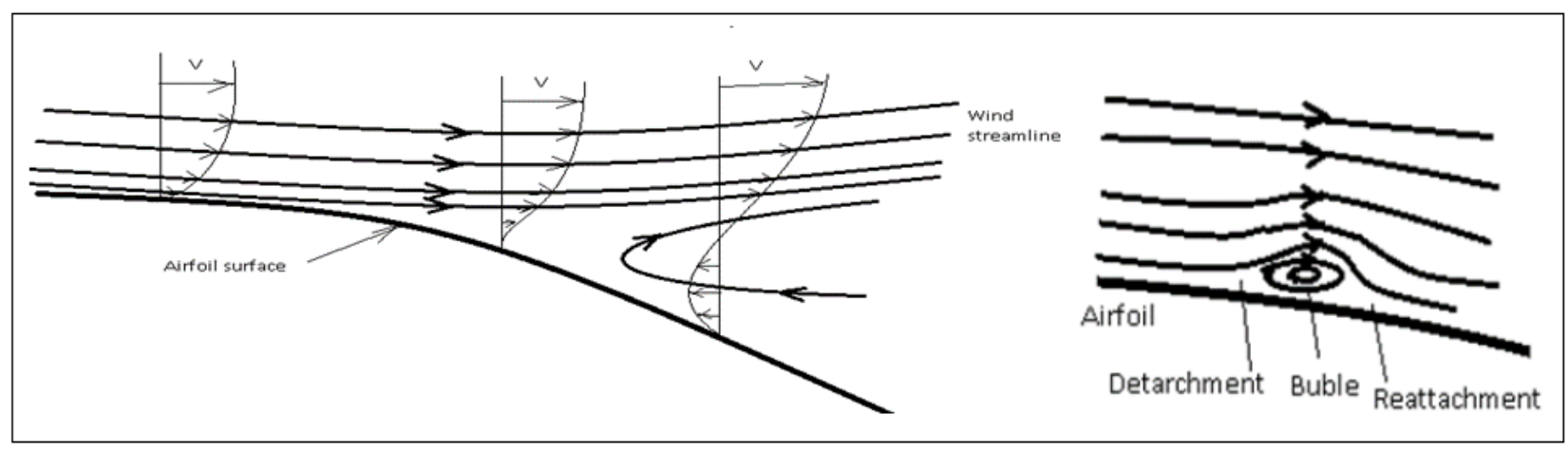

Figure 2. Velocity profile on separation. 
Separation in turbulent occurs when the angle of attack is very high. According to Hansen et al. (2014) a detached flow may however, reattach itself to form a bubble when the transition to turbulent occurs due to vortices (Fig 2). The use of turbine in the environmental conditions corresponding to its design Reynolds number can minimize the effect of LSB i.e. ambient Reynolds number must be equal to design Reynolds number. Ambient Reynolds number is the one derived from the local average wind speed at the area of installation.

On the other hand, the airfoil thickness affects the ambient Reynolds number. Ambient Reynolds number is found to increase with the increase in maximum thickness of the airfoil. For optimal lift to drag ratio, extremely low Re airfoil requires extremely low airfoil thickness (Clausen and Wood, 2000). According to Rehman et al. (2018), these findings have led to categorizing wind turbines as:

i. Low-speed wind turbine $\left(\operatorname{Re}<10^{3}\right)$

ii. Medium-speed wind turbine $\left(10^{3}<\operatorname{Re}<10^{5}\right)$

iii. High-speed wind turbine $\left(\operatorname{Re}>10^{5}\right)$

\section{DESIGN VS. AMBIENT REYNOLDS NUMBERS}

Flows under the same Reynolds numbers behave the same and vice versa (Manwell et al., 2009). High Reynolds number airfoil will perform well in high ambient Reynolds number than low ones. When designing Small Wind Turbine (SWT), normally airfoil is chosen from published airfoil data most of which were specifically designed for full scale aircraft and thus are high Reynolds number airfoils (Giguere and Selig, 1997). Consequently, most of commercial SWTs do not perform adequately in low wind speed regimes predominant in tropical equatorial regions.

For given airfoil parameters, different Reynolds numbers will lead to different lift/ drag ratios. Jain et al. (2015) in their study on NACA 0012 airfoil found that a change of Reynolds number from $1.5 \times 10^{5}$ to $1.0 \times 10^{5}$ at an angle of attack of $4^{0}$ decrements the lift coefficient by 1.2\%. Pires et al. (2016) performed wind tunnel test on high Reynolds number airfoil, DU00-W212 at different Reynolds number. It showed lowest lift to drag ratio at lower ambient Reynolds number as could be shown in figure 3.

According to investigation by Bak (2007) on Ris $\varnothing-B 1$ airfoil family, at Reynolds number of 200,000 the maximum lift to drag ratio was found to be 50 and power coefficient 0.53 but when changed to 900,000 , the lift to drag ratio became 180 and power coefficient 0.56 . For that matter, 
SWT designed for low wind speed regime must employ low Reynolds number airfoil in order to give a desired performance (Giguere and Selig, 1997).

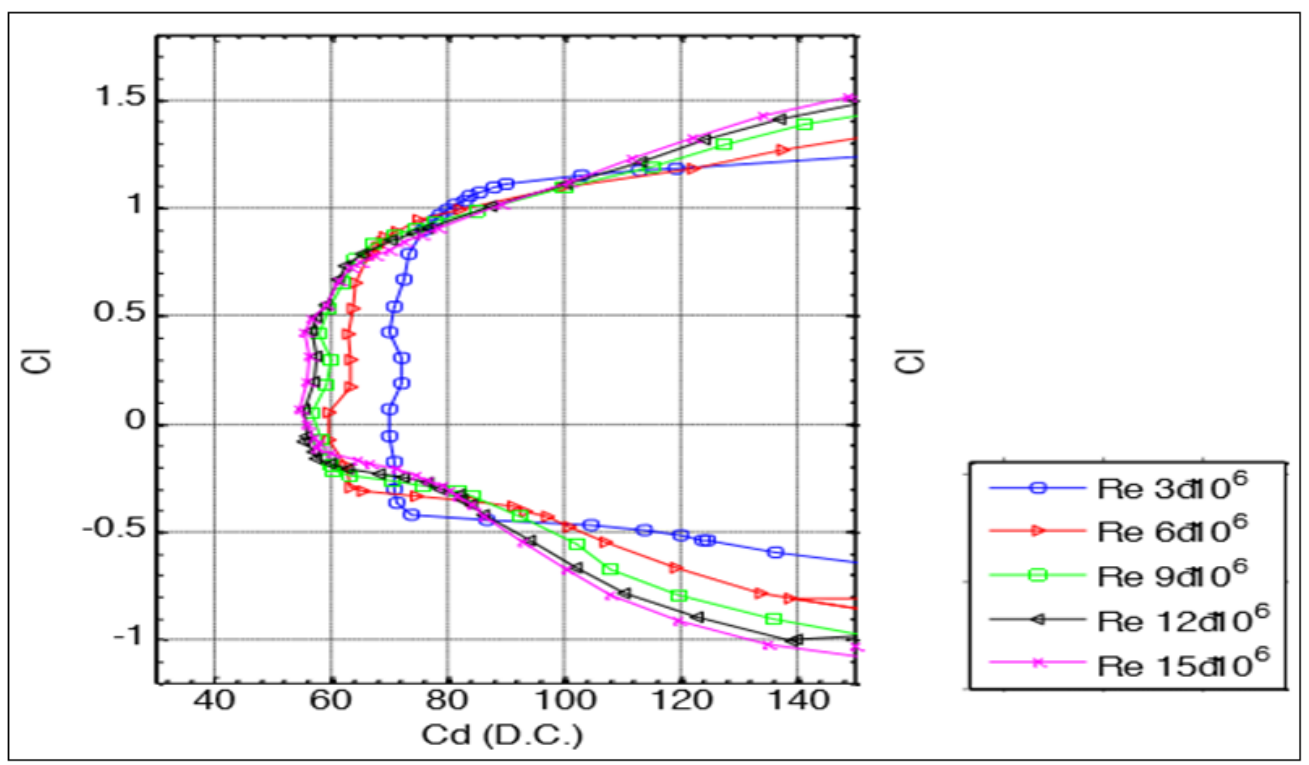

Figure 3. Lift coefficient against drag coefficient (Source: Pires et al., 2016).

However, the challenge has been the availability of low Reynolds number airfoil to correspond with low ambient Reynolds number encountered in tropical equatorial climate. Before publication, an airfoil is tested against design specifications. According to Hasan et al. (2017), airfoils are tested practically in a wind tunnel within a range of Reynolds numbers and surface conditions, but the wind tunnel technology cannot test very low Reynolds number. So, the available lowest Reynolds number airfoil dictates the lowest ambient Reynolds number SWT design that can be found in the market because of testing limitations. According to Airfoil tools (2020), the lowest available airfoil Reynolds number is 50,000. When it is chosen to design a SWT for the wind regime of ambient Reynolds number less than 50,000, the turbine would perform less than predicted. Moreover, the SWT will be more susceptible to stall due to LSB. Hung and Ngoc (2011) conducted experiments on NACA 0012 airfoil, at Reynolds number of orders $10^{5}$ there was no laminar separation noted for all angles of attack. However, at Reynolds number of 13,000, laminar separation was noticed.

According to Jahanmiri (2011), length, height and position of the bubble are influenced by changes in local ambient Reynolds number and the angle of attack. Higher Reynolds number decreases the length of the bubble. On the other hand, decrease in angle of attack below critical 
angle moves the bubble downstream towards trailing edge (Hansen et al., 2014; Genc and Kaynak, 2009). That is why some medium and large horizontal axis wind turbines are started by increasing pitch angle thereby decreasing angle of attack to avoid failure to starting due to stall. Other controls of LSB involve creating turbulent upstream because according to Jahanmiri (2011) it stabilizes the boundary layer. Genc and Kaynak (2009) investigation showed the early method for controlling LSB which was used in airplanes using the slats on the leading edge as turbulentors. The recent active method is blowing thereby creating turbulent upstream (Genc and Kaynak, 2009; Jahanmiri, 2011; Hasan et al., 2017).

On the other hand, drag-type wind turbine operates on the principle of conservation of momentum other than lift force as for airfoil bladed SWTs and thus are not affected by ambient Reynolds number (Kaltschmitt et al., 2013). Windmills such as American windmills are drag-type wind turbines, the most common locally manufactured wind turbines in tropical developing countries. They have advantage of having high torque and thus are commonly used for water pumping. However, they have poor power performance and thus not suitable for electricity generation (Mathew, 2004). One of the visited local small wind turbine manufacturer based in Dar es Salaam, Tanzania is used to display the drag-type wind turbine of his manufacturing together with the commercial lift type small wind turbine. The drag-type is seen to rotate with lower wind speed than the commercial ones. Observers and customers were being told that the latter is meant for European winds and not for tropical equatorial winds found in Tanzania, while the fact is that the latter which is designed for high Reynolds number suffers from stall as a result of LSBs in low wind speed.

\section{CONCLUSIONS}

Wind turbine performance decreases when the wind turbine is made to operate in ambient Reynolds number different from the design Reynolds number. The performance of low speed wind turbine could be maintained high by operating it in low local ambient Reynolds number i.e. low speed wind regime.

When the angle of attack is decreased, LSB moves downstream and thus may be eliminated completely. However, decrease in angle of attack may have a detrimental effect on power performance. So a trade-off which gives the highest energy output is essential during design. This 
paper recommends for a research which optimizes energy output by manipulating both the angle of attack and LSB.

No literature which shows the use of slats as turbulentor in wind turbine. It is probably difficult to implement in HAWT. In future an experiment is recommended to be conducted to see if using small parallel ridges on the airfoil surface between leading edge and point of maximum thickness can act as turbulentor without affecting the lift force or intended power coefficient. The occurrence of LBS has been a major hindrance in the optimization of low speed wind turbines.

\section{ACKNOWLEDGEMENTS}

We thank Energy and Petroleum project of the University of Dar es Salaam for their sponsoring the visit to various local manufactures which led to problem identification. We thank various individuals for their constructive critics which made the paper better. Finally, we appreciate for publication acceptance of our paper in the journal.

\section{CONFLICT OF INTERESTS}

No conflict of interests.

\section{REFERENCE}

Airfoil Tools. 2020. Airfoil Database Search. Available at: <http://airfoiltools.com/search/index?

Bak, C. 2007. Sensitivity of Key Parameters in Aerodynamic Wind Turbine Rotor Design on Power and Energy Performance. Journal of Physics: Conference Series 75: 012008

Bertin J. J \& Cummings, R. M. 2009. Aerodynamics for Engineers. $5^{\text {th }}$ Edition, Pearson Education, Inc., USA, pp. 640-643.

Clausen, P. D \& Wood, D. H. 2000. Recent Advances in Small Wind Turbine Technology. Wind Engineering, 24(3): 189- 201

Genç, M. S \& Kaynak, Ü. 2009. Control of Laminar Separation Bubble over a NACA2415 Aerofoil at Low $\operatorname{Re}$ Transitional Flow Using Blowing/Suction. $13^{\text {th }}$ International Conference on Aerospace Sciences \& Aviation Technology, ASAT- 13.

Giguere, P \& Selig, M. S. 1997. Low Reynolds number Airfoil for Small Horizontal Axis Wind Turbine. Wind Energy, Sage publications, UK, 21(6): 367-380. 
Hasan, M. M. 2017. Design and Performance Analysis of Small Scale Horizontal Axis Wind Turbine for Nano Grid Application. Master of Science Thesis, Georgia Southern University https://digitalcommons.georgiasouthern.edu/etd

Hansen, K. L., Kelso, R. M., Choudhry, A \& Arjomandi, M. 2014. Laminar Separation Bubble Effect on the Lift Curve Slope of an Airfoil. 19th Australasian Fluid Mechanics Conference, Melbourne.

Hepperle, M. 2018. Reynodes number calculation. www.mhaerotools.de/airfoils/howdoi.htm accessed on 20/03/2018 at 1306.

Hung, N \& Ngoc, H. 2011. Experimental study of laminar separation phenomenon combining with numerical calculations. Vietnam Journal of Mechanics, 33: 95-104.

Incropera, F. P., Dewitt, D. P., Bergman, T. L \& Lavine, A. S. 2007. Fundamentals of Heat and Mass Transfer. $6^{\text {th }}$ Edition, John Wiley \& Sons, Inc., USA, pp. 360-365.

Jahanmiri, M. 2011. Laminar Separation Bubble: Its Structure, Dynamics and Control. Research report 2011:06, https://core.ac.uk/download/pdf/70588164.pdf accessed 05/06/2017 at 2314.

Jain, S., Sitaram, N \& Krishnaswamy, S. 2015. Effect of Reynolds Number on Aerodynamics of Airfoil with Gurney Flap. International Journal of Rotating Machinery, Article ID 628632, http://dx.doi.org/10.1155/2015/628632 accessed 24/01/2017 at 0827

Kaltschmitt, M., Themelis, N. J., Bronicki, L. Y., Söder, L \& Veg, L. A. 2013. Renewable Energy Systems. Springer, New York, pp. 696-711.

Kim, Y \& Al-Abadi, A. 2016. Strategic Blade Shape Optimization for Aerodynamic Performance Improvement of Wind Turbines. Proceedings of ASME Turbomachinery Technical Conference and Exposition, Seoul, South Korea, GT 2016-56836.

Manwell, J.F., McGowan, J.G \& Rogers, A.L. 2009. Wind Energy Explained: Theory, Design and Application. $2^{\text {nd }}$ Edition, John Wiley \& Sons Ltd., UK, pp. 103-115.

Mathew, S. 2006. Wind Energy, Fundamentals, Resource Analysis and Economics. SpringerVerlag Berlin Heidelberg, Netherlands, pp. 23-27.

Pires, O., Munduate, X., Ceyhan, O., Jacobs, M \& Snel, H. 2016. Analysis of high Reynolds numbers effects on a wind turbine airfoil using 2D wind tunnel test data. Journal of Physics: Conference Series, 753022047 (doi:10.1088/1742-6596/753/2/022047). 
Rehman, S., Alam, M. M., Alhems, L. M \& Rafique, M. M. 2018. Horizontal Axis Wind Turbine Blade Design Methodologies for Efficiency Enhancement. Energies, 11(3): 506, (doi:10.3390/en11030506).

Sabri, L. A., Mohammed, S. A \& Issa, M. I. 2017. Design of SCADA System for Oil Pipeline Control Using LabVIEW. Al-Nahrain Journal for Engineering Sciences (NJES), 20(3): 600614.

Schaffarczyk, A. P. 2014. Introduction to Wind Turbine Aerodynamics. Springer-Verlag Berlin Heidelberg, pp. 42-52.

Shah, H., Mathew, S \& Lim, C. M. 2014. A Novel Low Reynolds Number Airfoil Design for Small Horizontal Axis Wind Turbines. Wind Engineering, 38(4): 377-392.

Yousefi, K \& Razeghi, A. 2018. Determination of the Critical Reynolds Number for Flow over Symmetric NACA Airfoils. AIAA 2018-0818, (doi: 10.2514/6.2018-0818). 